\title{
The Prognostic Value of Myocardial Injury in COVID-19 Patients and Associated Characteristics
}

jian he

National Center for Cardiovascular Diseases China: Chinese Academy of Medical Sciences \& Peking Union Medical College

Fuwai Hospital

Bicheng Zhang

Renmin Hospital of Wuhan University: Wuhan University Renmin Hospital

Quan Zhou

Renmin Hospital of Wuhan University: Wuhan University Renmin Hospital

Wenjing Yang

National Center for Cardiovascular Diseases China: Chinese Academy of Medical Sciences \& Peking Union Medical College

Fuwai Hospital

Jing Xu

National Center for Cardiovascular Diseases China: Chinese Academy of Medical Sciences \& Peking Union Medical College

Fuwai Hospital

Tingting Liu

Renmin Hospital of Wuhan University: Wuhan University Renmin Hospital

Haijun Zhang

Renmin Hospital of Wuhan University: Wuhan University Renmin Hospital

Zhiyong Wu

Renmin Hospital of Wuhan University: Wuhan University Renmin Hospital

Dong Li

Renmin Hospital of Wuhan University: Wuhan University Renmin Hospital

\section{Qing Zhou}

Renmin Hospital of Wuhan University: Wuhan University Renmin Hospital

Jie Yan

Renmin Hospital of Wuhan University: Wuhan University Renmin Hospital

\section{Cuizhen Zhang}

Renmin Hospital of Wuhan University: Wuhan University Renmin Hospital

\section{Robert G. Weiss}

Johns Hopkins School of Medicine: Johns Hopkins University School of Medicine

\section{Guanshu Liu}

Johns Hopkins School of Medicine: Johns Hopkins University School of Medicine

\section{Zhongzhao Teng}

Cambridge University: University of Cambridge

\section{Arlene Sirajuddin}

National Heart Lung and Blood Institute

\section{Haiyan Qian}

National Center for Cardiovascular Diseases China: Chinese Academy of Medical Sciences \& Peking Union Medical College Fuwai Hospital

\section{Shihua Zhao}

National Center for Cardiovascular Diseases China: Chinese Academy of Medical Sciences \& Peking Union Medical College Fuwai Hospital 
Andrew E. Arai

National Heart Lung and Blood Institute

Minjie Lu ( $\nabla$ coolkan@163.com )

National Center for Cardiovascular Diseases China: Chinese Academy of Medical Sciences \& Peking Union Medical College Fuwai Hospital

Xiaoyang Zhou

Renmin Hospital of Wuhan University: Wuhan University Renmin Hospital

\section{Research article}

Keywords: Coronavirus disease 2019, prognosis, myocardial injury, clinical characteristics

Posted Date: February 19th, 2021

DOI: https://doi.org/10.21203/rs.3.rs-251810/v1

License: (c) (i) This work is licensed under a Creative Commons Attribution 4.0 International License. Read Full License

Version of Record: A version of this preprint was published at Immunity, Inflammation and Disease on July 9th, 2021. See the published version at https://doi.org/10.1002/iid3.484. 


\section{Abstract}

Background: Since December 2019, Coronavirus disease 2019 (COVID-19) has emerged as an international pandemic. COVID-19 patients with myocardial injury might need special attention. However, understanding on this aspect remains unclear. This study aimed to illustrate clinical characteristics and the prognostic value of myocardial injury to COVID-19 patients.

Methods: This retrospective, single-center study finally included 304 hospitalized COVID-19 cases confirmed by real-time RT-PCR from January 11 to March 25, 2020. Myocardial injury was determined by serum high-sensitivity troponin I (Hs-Tnl). The primary endpoint was COVID-19 associated mortality.

Results: Of 304 COVID-19 patients (median age, 65 years; $52.6 \%$ males), 88 patients (27.3\%) died (61 patients with myocardial injury, 27 patients without myocardial injury on admission). COVID-19 patients with myocardial injury had more comorbidities (hypertension, chronic obstructive pulmonary disease, cardiovascular disease, and cerebrovascular disease); lower lymphocyte counts, higher C-reactive protein (CRP, median, $84.9 \mathrm{vs} 28.5 \mathrm{mg} / \mathrm{L}, \mathrm{p}<0.001$ ), procalcitonin levels (median, $0.29 \mathrm{vs} 0.06 \mathrm{ng} / \mathrm{ml}$, $\mathrm{p}<0.001$ ), inflammatory and immune response markers; more frequent need for noninvasive ventilation, invasive mechanical ventilation; and was associated with higher mortality incidence (hazard ratio, $\mathrm{HR}=7.02,95 \%$ confidence interval, $\mathrm{Cl}, 4.45-11.08$, $\mathrm{p}<0.001)$ than those without myocardial injury. Myocardial injury $(\mathrm{HR}=4.55,95 \% \mathrm{Cl}, 2.49-8.31, \mathrm{p}<0.001)$, senior age, CRP levels, and novel coronavirus pneumonia (NCP) types on admission were independent predictors to mortality in COVID-19 patients.

Conclusions: COVID patients with myocardial injury on admission is associated with more severe clinical presentation and biomarkers. Myocardial injury and higher HsTNI are both strongest independent predictors to COVID related mortality after adjusting confounding factors. In addition, senior age, CRP levels and NCP types are also associated with mortality.

Trial registration: Not applicable.

\section{Background}

Coronavirus disease 2019 (COVID-19) is a newly recognized infection which was first reported in Wuhan, China [1]. Since the beginning of the outbreak, COVID-19 has emerged as a pandemic globally [2], and the number of cases is rising at an exponential rate [3]. As of November 15, 2020, there have been a total of more than 53,766,728 laboratory-confirmed cases of COVID-19 globally, and it poses a great threat to public health in the world as evidenced by $1,308,975$ deaths [4]. COVID-19 is caused by the severe acute respiratory syndrome coronavirus-2 (SARS-CoV-2). The clinical spectrum appears to be very wide, including asymptomatic infection, mild upper respiratory disease, and severe viral pneumonia with respiratory failure, and even death [5]. The condition of some patients with COVID-19 may deteriorate rapidly, particularly in older patients with underlying comorbidities including cardiovascular disease [6]. Furthermore, SARS-CoV-2 can affect the cardiovascular system in multiple ways, increasing morbidity in patients with potential cardiovascular disease and causing myocardial injury and dysfunction [3]. High-sensitivity troponin I (Hs-Tnl) provides the potential to earlier identify myocardial injury and assists treatment [7]. Some studies provide insights into the incidence of cardiac complications associated with SARS-CoV-2 [8, 9], while imaging manifestations, cytokine levels and the prognostic value of cardiovascular risk factors in COVID-19 patients are poorly understood. We aimed to comprehensively define clinical characteristics, laboratory results, outcomes, and management strategies of COVID-19 patients, then to find whether there is an association of myocardial injury and other biomarkers with mortality. This study may also provide clues to potential mechanisms associated with myocardial injury.

\section{Methods}

\section{Study Design And Patients}

In this study, we retrospectively enrolled 320 COVID-19 patients admitted to the Renmin Hospital of Wuhan University from January 11, 2020 to March 25, 2020 with approval from the Research Ethics Committee of the Renmin Hospital of Wuhan University, Wuhan, China (approval number: WDRY2020-K038). 16 cases without significant biomarkers, including Hs-Tnl and creatinine kinase-myocardial band (CK-MB) levels, were excluded. Thus, a total of 304 patients were finally included in the study. 
The confirmed diagnosis of COVID-19 was defined as a positive result by using real-time reverse-transcriptase polymerase-chainreaction (RT-PCR) detection for routine nasal and pharyngeal swab specimens or anti-SARS-CoV-2 antibody assay.

\section{Data Collection}

The demographic characteristics, clinical data, and results of cardiac biomarkers were obtained from the hospital's electronic medical records according to previously designed standardized data collection forms. The date of symptom onset, initial diagnosis of COVID-19, and death were recorded. The clinical features of symptoms and signs and comorbidities were collected on admission. Laboratory analyses included complete blood count, hepatic function, kidney function, coagulation function, Creactive protein (CRP), lactate dehydrogenase $(\mathrm{LDH})$, myocardial enzymes, procalcitonin (PCT), electrolytes test, and status of other viral infection. Cardiac biomarkers including N-terminal pro-B-type natriuretic peptide (NT-proBNP), Hs-Tnl, CK-MB, and myoglobin were measured on admission and during hospitalization. Routine bacterial and fungal examinations were also performed. Radiologic assessments included digital radiography (DR) and/or computed tomography (CT). Two researchers collected and checked the final database.

\section{Definitions}

Fever was defined as an axillary temperature of $37.5^{\circ} \mathrm{C}$ or higher [10]. The date of onset was defined as the day when any symptom was noticed. Myocardial injury was defined as serum levels of Hs-Tnl were above the 99th percentile upper reference limit at admission $(0.04 \mathrm{ng} / \mathrm{mL}$, measured in the laboratory of Renmin Hospital of Wuhan University) [11]. The novel coronavirus pneumonia (NCP) types (mild, common, severe, critically severe) was defined according to the diagnostic and treatment programme for SARS-CoV-2 issued by Chinese National Health Committee (version 7) [12]. The primary end point was COVID-19 associated death. Hospital discharge was allowed after the relieved clinical symptoms, normal body temperature for at least three days, significant improvement in radiological findings, and at least two consecutive negative results shown by RT-PCR for COVID19 [12].

\section{Statistical analysis}

Categorical variables are shown as frequency rates or percentages. Continuous variables as mean \pm standard deviation (SD) or median (interquartile range [IQR]) as appropriate. Student's $t$ test, Mann Whitney test, $\chi^{2}$ test or Fisher exact test was used where appropriate to assess the difference between different cohorts. The Pearson correlation coefficient and Spearman rank correlation coefficient were used for correlation analysis. Survival curves were plotted using the Kaplan-Meier method between patients with and without myocardial injury. Univariate and multivariate Cox regression models were used to determine the independent risk factors for death in hospital. The NCP types was listed as rank variable. Statistical analysis was performed with SPSS, version 21.0 (IBM, USA). A two-sided $\mathrm{P}<0.05$ was considered statistically significant.

\section{Results}

A total of 304 hospitalized patients with COVID-19 were consecutively enrolled in our study, including 96 patients (31.6\%) with myocardial injury and 208 patients (68.4\%) without myocardial injury. The demographic and clinical characteristics of the patients are listed in Table 1. On admission, over half of the COVID-19 patients $(198,65.1 \%)$ had comorbidities, including hypertension (42.8\%), diabetes (16.4\%), and cardiovascular disease (16.1\%), like coronary artery disease (10.5\%), arrythmia (4.3\%), and cardiomyopathy (0.7\%). COVID-19 patients with myocardial injury were older, more males, and more likely to have preexisting comorbidities, and were associated with more severe presentation (critically severe, $26.0 \%$ vs $7.2 \%$, all $p<0.001$ ). 
Table 1

Clinical characteristics and complications of COVID-19 patients

\begin{tabular}{|c|c|c|c|c|}
\hline \multirow[t]{2}{*}{ Characteristic } & \multirow{2}{*}{$\begin{array}{l}\text { All patients } \\
(n=304)\end{array}$} & \multicolumn{2}{|l|}{ Myocardial injury } & \multirow[t]{2}{*}{$P$ value } \\
\hline & & With $(n=96)$ & Without $(n=208)$ & \\
\hline Male (\%) & $160(52.6)$ & $61(63.5)$ & $99(47.6)$ & 0.010 \\
\hline Age, median (IQR) & $65.0(54.0-74.0)$ & $70.5(60.1-79.0)$ & $62.0(52.0-69.0)$ & $<0.001$ \\
\hline \multicolumn{5}{|l|}{ NCP types, (\%) } \\
\hline Mild / Common & $117(38.5)$ & $22(22.9)$ & $95(45.7)$ & \multirow[t]{3}{*}{$<0.001$} \\
\hline Severe & $147(48.4)$ & $49(51.0)$ & $98(47.1)$ & \\
\hline Critically severe & $40(13.2)$ & $25(26.0)$ & $15(7.2)$ & \\
\hline Comorbidities, (\%) & $198(65.1)$ & $76(79.2)$ & $122(58.7)$ & $<0.001$ \\
\hline Diabetes & $50(16.4)$ & $17(17.7)$ & $33(15.9)$ & 0.687 \\
\hline Hypertension & $130(42.8)$ & $52(54.2)$ & $78(37.5)$ & 0.006 \\
\hline COPD & $21(6.9)$ & $12(12.5)$ & $9(4.3)$ & 0.009 \\
\hline Cardiovascular disease & $49(16.1)$ & $27(28.1)$ & $22(10.6)$ & $<0.001$ \\
\hline Coronary artery disease & $32(10.5)$ & $18(18.8)$ & $14(6.7)$ & 0.002 \\
\hline Cerebrovascular disease & $21(6.9)$ & $13(13.5)$ & $8(3.8)$ & 0.002 \\
\hline Kidney disease & $12(3.9)$ & $4(4.2)$ & $8(3.8)$ & 1 \\
\hline Hepatic disease & $8(2.6)$ & $3(3.1)$ & $5(2.4)$ & 0.711 \\
\hline Cancer, Auto-immune disease & $32(10.5)$ & $11(11.5)$ & $21(10.1)$ & 0.719 \\
\hline
\end{tabular}

On admission, the median leukocyte counts, neutrophil counts, platelet counts, hemoglobin, immunoglobulin $\mathrm{G}(\lg \mathrm{g})$, IgM, $\lg \mathrm{A}$ and complement 3 (C3) levels were all within the normal range in both groups (Table 2). However, significant differences were noted in neutrophil and platelet counts between the two groups $(p<0.001)$. Comparing with patients without myocardial injury, patients with myocardial injury had even lower CD3, CD4, CD8 counts, higher IL-6, CRP, and PCT levels (all $p<0.001$ ), and CD3, CD4, CD8 counts showed strong correlations $(R>0.77)$ with lymphocytes counts and moderate correlations $(R:-0.39 \sim-0.45)$ with $C R P$ levels (Fig. 1). Log-transformed serum Hs-Tnl levels in patients with COVID-19 correlated significantly with both log-transformed serum NT-proBNP levels $(\beta=0.37, p<0.001)$ and serum urea nitrogen levels $(\beta=4.23, p<0.001)$ (Fig. 2). 
Table 2

Laboratory examinations and radiographic presentation in COVID-19 patients

\begin{tabular}{|c|c|c|c|c|c|}
\hline \multirow[t]{2}{*}{ Characteristic } & \multirow{2}{*}{$\begin{array}{l}\text { Normal } \\
\text { range }\end{array}$} & \multirow{2}{*}{$\begin{array}{l}\text { All patients } \\
(n=304)\end{array}$} & \multicolumn{2}{|l|}{ Myocardial injury } & \multirow{2}{*}{$\begin{array}{l}P \\
\text { value }\end{array}$} \\
\hline & & & With $(n=96)$ & Without $(n=208)$ & \\
\hline \multicolumn{6}{|c|}{ Immunologic markers, median (IQR) } \\
\hline Leucocyte counts, $10^{9} / \mathrm{L}$ & $3.5-9.5$ & $6.3(4.3-8.1)$ & $7.1(5.0-10.1)$ & $6.0(4.4-7.8)$ & 0.058 \\
\hline Neutrophil counts, $10^{9} / \mathrm{L}$ & $1.8-6.3$ & $4.9(3.0-7.4)$ & $6.7(4.2-10.3)$ & $4.4(2.8-6.4)$ & $\hat{0} .001$ \\
\hline $\begin{array}{l}\text { Lymphocyte } \\
\text { counts, } 10^{9} / \mathrm{L}\end{array}$ & $1.1-3.2$ & $0.9(0.6-1.3)$ & $0.6(0.4-1.0)$ & $1.0(0.7-1.3)$ & $\hat{0.001}$ \\
\hline Platelet counts, $10^{9} / \mathrm{L}$ & $130-175$ & $200.0(145.0-258.0)$ & 151.5(105.0-237.2) & $210.0(165.5-264.0)$ & $\dot{0} 001$ \\
\hline Hemoglobin, $g / L$ & $125-350$ & $123.0(111.3-136.0)$ & $122.0(110.0-137.0)$ & $123.0(113.0-135.2)$ & 0.872 \\
\hline CD 3 counts, /uL & $723-2737$ & $502.0(275.8-765.0)$ & $317.5(177.3-591.0)$ & $539.5(324.0-862.5)$ & $\hat{0} .001$ \\
\hline CD 4 counts, /uL & $404-1612$ & $287(161.5-464.5)$ & $193.5(98.8-322.8)$ & $340.5(201.8-528.3)$ & $\dot{0} 001$ \\
\hline CD 8 counts, /uL & $220-1129$ & $152.0(73.0-278.0)$ & $77(40.8-161.5)$ & $191.0(106.0-304.0)$ & $\hat{0} .001$ \\
\hline CD4/CD8 ratio & $0.9-2.0$ & $1.8(1.3-1.7)$ & $2.0(1.3-3.4)$ & $1.8(1.3-2.7)$ & 0.127 \\
\hline $\lg G, g / L$ & $7.0-16.0$ & $12.3(10.2-15.4)$ & $13.7(11.2-16.6)$ & $11.7(9.6-14.6)$ & 0.003 \\
\hline $\lg M, g / L$ & $0.4-2.3$ & $1.0(0.7-1.3)$ & $1.0(0.7-1.3)$ & $1.0(0.7-1.2)$ & 0.143 \\
\hline $\lg A, g / L$ & $0.7-4.0$ & $2.4(1.8-3.3)$ & $2.8(2.1-3.8)$ & $2.2(1.7-3.0)$ & 0.002 \\
\hline $\mathrm{C} 3, \mathrm{~g} / \mathrm{L}$ & $0.9-1.8$ & $1.0(0.9-1.1)$ & $1.0(0.8-1.1)$ & $1.0(0.9-1.2)$ & $\hat{0.001}$ \\
\hline CK-MB, ng/mL & $0-5$ & $1.2(0.7-2.6)$ & $4.2(1.9-8.3)$ & $0.9(0.7-1.5)$ & $\dot{0} .001$ \\
\hline \multicolumn{6}{|c|}{ Cardiac, hepatic and kidney injury markers, median (IQR) } \\
\hline Myoglobin, ug/L & $0-110$ & $49.1(27.4-130.9)$ & $177.4(82.5-765.7)$ & $35.0(25.0-59.7)$ & $\dot{0} .001$ \\
\hline $\mathrm{Hs}-\mathrm{TNI}, \mathrm{ng} / \mathrm{mL}$ & $0-0.04$ & $\begin{array}{l}<0.006(<0.006- \\
0.068)\end{array}$ & $0.22(0.09-1.83)$ & $\begin{array}{l}<0.006(<0.006- \\
0.011)\end{array}$ & $\dot{0} 001$ \\
\hline NT-proBNP\#, pg/mL & $0-300$ & $285.8(86.7-835.8)$ & $\begin{array}{l}799.7(267.7- \\
1719.0)\end{array}$ & $220.1(54.0-456.5)$ & $\dot{0} 001$ \\
\hline NT-proBNP*, pg/mL & $0-300$ & $647.8(237.2-1996.3)$ & $\begin{array}{l}2543.0(953.0- \\
9022.0)\end{array}$ & 389.0 (141.0-1046.0) & $\dot{0.001}$ \\
\hline $\mathrm{LDH}, \mathrm{U} / \mathrm{L}$ & $100-300$ & $266(202.8-413.3)$ & $433.5(306.5-677.5)$ & $221.0(188.0-284.0)$ & $\hat{0} .001$ \\
\hline$A L T, U / L$ & $9-50$ & $24.0(17.0-46.0)$ & $27.0(18.0-48.0)$ & $24.0(16.8-43.3)$ & 0.662 \\
\hline
\end{tabular}

P-values are calculated by Student's t-test, Mann-Whitney U test, or $\chi^{2}$ test as appropriate. ${ }^{*}$, NT-proBNP levels on admission; *, NT-proBNP levels during hospitalization. Abbreviation: IQR, interquartile range; CD, Cluster of Differentiation; Ig,

Immunoglobulin; C3, Complement 3; IL-6, interleukin 6; PT, Prothrormbin time; APTT, Active Partial Thromboplastin Time; CKMB, Creatinine kinase-myocardial band; Hs-TNI, High-sensitivity troponin I; NT-proBNP, N-terminal pro-B-type natriuretic peptide; PCT, Procalcitonin; LDH, Lactate Dehydrogenase; ALT, Alanine aminotransferase; AST, Aspartate aminotransferase; ALP, alkaline phosphatase; ALB, Albumin; ECG, electrocardiogram. 


\begin{tabular}{|c|c|c|c|c|c|}
\hline \multirow[t]{2}{*}{ Characteristic } & \multirow{2}{*}{$\begin{array}{l}\text { Normal } \\
\text { range }\end{array}$} & \multirow{2}{*}{$\begin{array}{l}\text { All patients } \\
(n=304)\end{array}$} & \multicolumn{2}{|l|}{ Myocardial injury } & \multirow{2}{*}{$\begin{array}{l}P \\
\text { value }\end{array}$} \\
\hline & & & With $(n=96)$ & Without $(n=208)$ & \\
\hline AST, U/L & $15-40$ & $27.0(19.0-43.5)$ & $42.0(24.0-65.0)$ & $23.5(17.3-32.0)$ & $\begin{array}{l}< \\
0.001\end{array}$ \\
\hline ALP, U/L & $90-130$ & $71.0(56.4-94.0)$ & $75.0(58.8-105.0)$ & $69.0(56.0-90.3)$ & 0.219 \\
\hline ALB, $g / L$ & $40-55$ & $37.0(33.5-40.0)$ & $33.8(30.1-37.1)$ & $38.7(35.8-41.0)$ & $\begin{array}{l}< \\
0.001\end{array}$ \\
\hline Urea, mmol/l & $3.6-9.5$ & $5.4(3.8-9.0)$ & $9.5(5.4-19.4)$ & $4.6(3.5-6.5)$ & $\begin{array}{l}< \\
0.001\end{array}$ \\
\hline Creatinine, $\mathrm{mmol} / \mathrm{L}$ & $57-111$ & $58.0(48.0-79.0)$ & $71.0(53.0-126)$ & $56.0(46.0-70.8)$ & $\begin{array}{l}< \\
0.001\end{array}$ \\
\hline Potassium, mmol/L & $3.5-5.5$ & $4.2(3.8-4.5)$ & $4.1(3.6-4.6)$ & $4.3(3.9-4.5)$ & 0.255 \\
\hline Sodium, $\mathrm{mmol} / \mathrm{L}$ & $135-155$ & $142.0(139.0-146.0)$ & $141.0(138.0-146.0)$ & $142.0(139.0-146.0)$ & 0.542 \\
\hline \multicolumn{6}{|c|}{ Inflammation markers, median (IQR) } \\
\hline PCT, ng/mL & $<0.1$ & $0.10(0.05-0.32)$ & $0.29(0.10-1.09)$ & $0.06(0.04-0.14)$ & $\begin{array}{l}< \\
0.001\end{array}$ \\
\hline C-reactive protein, $\mathrm{mg} / \mathrm{L}$ & $0-10$ & $51.3(10.9-104.0)$ & $84.9(53.7-173.8)$ & $28.5(5.7-82.2)$ & $\begin{array}{l}< \\
0.001\end{array}$ \\
\hline $\mathrm{IL}-6, \mathrm{pg} / \mathrm{mL}$ & $<10$ & $10.5(6.1-26.5)$ & $23.5(10.7-98.1)$ & $9.0(5.8-20.6)$ & $\begin{array}{l}< \\
0.001\end{array}$ \\
\hline \multicolumn{6}{|c|}{ Coagulation markers, median (IQR) } \\
\hline PT, sec & $9-13$ & $12.4(11.5-13.5)$ & $13.4(12.2-14.4)$ & $12.1(11.3-13.1)$ & $\hat{0} .001$ \\
\hline APTT, sec & $25-31.3$ & $28.6(26.2-31.5)$ & $29.2(27.7-33.2)$ & $28.2(25.9-31.0)$ & $\hat{0} .001$ \\
\hline D-dimer, mg/L & $0-0.55$ & $2.5(0.7-13.8)$ & $7.0(1.9-21.7)$ & $1.6(0.6-8.2)$ & $\hat{0} .001$ \\
\hline \multicolumn{6}{|c|}{$\begin{array}{l}\text { P-values are calculated by Student's t-test, Mann-Whitney U test, or } \chi^{2} \text { test as appropriate. \#, NT-proBNP levels on admission; *, } \\
\text { NT-proBNP levels during hospitalization. Abbreviation: IQR, interquartile range; CD, Cluster of Differentiation; Ig, } \\
\text { Immunoglobulin; C3, Complement 3; IL-6, interleukin 6; PT, Prothrormbin time; APTT, Active Partial Thromboplastin Time; CK- } \\
\text { MB, Creatinine kinase-myocardial band; Hs-TNI, High-sensitivity troponin l; NT-proBNP, N-terminal pro-B-type natriuretic } \\
\text { peptide; PCT, Procalcitonin; LDH, Lactate Dehydrogenase; ALT, Alanine aminotransferase; AST, Aspartate aminotransferase; } \\
\text { ALP, alkaline phosphatase; ALB, Albumin; ECG, electrocardiogram. }\end{array}$} \\
\hline
\end{tabular}

Regarding the cardiac, hepatic, renal and coagulation function (Table 2), patients with COVID-19 had increased Hs-Tnl, PCT and Ddimer levels on admission and NT-proBNP during hospitalization compared to the normal reference values. Patients with myocardial injury showed elevated myoglobin, $L D H, P C T$, and D-dimer levels (all $p<0.001$ ) compared to patients without myocardial injury. Figure 2 shows the dynamic change of NT-proBNP of COVID-19 patients from admission to hospitalization.

The Imaging manifestations of the 304 patients with COVID-19 were listed in table S1. 135 (44.4\%) underwent examination of electrocardiogram (ECG) after admission, and 83 of 135 ECGs (61.5\%) indicated cardiac abnormalities, including T-wave depression and inversion, ST-segment depression, atrioventricular block, and the complex abnormality. 34 (11.2\%) underwent examination with echocardiography and $27(79.4 \%)$ of patients showed abnormalities, and the more common abnormalities are cardiac diastolic dysfunction, and complex echo abnormalities (tricuspid regurgitation). All patients underwent CT or DR examinations and 221 (72.6\%) patients presented with pulmonary abnormalities including ground-glass opacities or consolidation (Fig S1). There was no statistically significant difference in the other imaging findings between patients with or without myocardial injury, only the patients with complex echo abnormalities had significant difference. 
Oxygen treatment was provided to $206 / 304(67.8 \%)$ patients. $62(20.4 \%)$ patients received noninvasive ventilation, and $10.2 \%$ patients (31 patients) were placed in mechanical ventilation. The proportion treated with antiviral therapy was the highest (304, $100 \%)$, followed by high dose glucocorticoids therapy (142, 46.7\%), intravenous immunoglobulin therapy (122, 40.1\%), antibiotic therapy $(118,38.8 \%)$ and hemoperfusion $(20,6.6 \%)$. Only 8 patients $(2.6 \%)$ among all participants were given plasmapheresis therapy. Overall, 100 patients $(27.3 \%)$ had kidney injury during hospitalization, and 65 patients $(21.4 \%)$ had hepatic injury.

Compared with those without myocardial injury, more COVID-19 patients with myocardial injury required oxygen inhalation; noninvasive ventilation, invasive mechanical ventilation, antibiotic treatment and hemoperfusion therapy (all $p<0.001)($ Table 3 ). However, intravenous immunoglobulin treatment $(24.0 \%$ vs $47.6 \%, \mathrm{P}<0.001)$ was lower in patients with myocardial injury. The comparison of typical managements, comorbidities, complications and time duration from symptom onset to death were made in 20 random patients with myocardial injury or not (Fig. 3), which tells us that myocardial injury may be associated with more severe presentation.

Table 3

Managements and clinical outcomes of COVID-19 patients

\begin{tabular}{|c|c|c|c|c|}
\hline \multirow[t]{2}{*}{ Characteristic } & \multirow{2}{*}{$\begin{array}{l}\text { All patients } \\
(n=304)\end{array}$} & \multicolumn{2}{|c|}{ Myocardial injury } & \multirow[t]{2}{*}{$P$ value } \\
\hline & & With $(n=96)$ & Without $(n=208)$ & \\
\hline \multicolumn{5}{|l|}{ Managements, n (\%) } \\
\hline Oxygen inhalation & $206(67.8)$ & $85(88.5)$ & $121(58.2)$ & $<0.001$ \\
\hline Noninvasive ventilation & $62(20.4)$ & $35(36.5)$ & $27(13.0)$ & $<0.001$ \\
\hline Invasive mechanical ventilation & $31(10.2)$ & $20(20.8)$ & $11(5.3)$ & $<0.001$ \\
\hline Immunoglobulin & $122(40.1)$ & $23(24.0)$ & $99(47.6)$ & $<0.001$ \\
\hline Antiviral & $304(100)$ & $96(100)$ & $208(100)$ & - \\
\hline Antibiotic & $118(38.8)$ & $65(67.7)$ & $53(25.5)$ & $<0.001$ \\
\hline Glucocorticoids & $142(46.7)$ & $44(45.8)$ & $98(47.1)$ & 0.835 \\
\hline Hemoperfusion & $20(6.6)$ & $12(12.5)$ & $8(3.8)$ & 0.005 \\
\hline Plasmapheresis & $8(2.6)$ & $5(5.2)$ & $3(1.4)$ & 0.114 \\
\hline \multicolumn{5}{|l|}{ Clinical outcomes, n (\%) } \\
\hline Death & $88(27.3)$ & $61(63.5)$ & $27(13.0)$ & \multirow[t]{3}{*}{0.005} \\
\hline In hospital & $83(28.9)$ & $11(11.5)$ & $72(34.6)$ & \\
\hline Discharge & $133(43.8)$ & $24(25.0)$ & $109(52.4)$ & \\
\hline
\end{tabular}

During the median durations for about 45.4 days from onset of symptoms to follow-up (range:3-84 days), a total of 88/304 patients (27.3\%) died, among which 61 patients had myocardial injury, while 27 patients didn't have myocardial injury (Table 3). In the univariable analysis, the mortality rate was significantly higher in patients with myocardial injury $(63.5 \%$ vs $13.0 \%, P<0.001$, Table 3). The Kaplan-Meier survival curves indicate significant survival differences between the patients with or without myocardial injury $(p<0.001$, Fig. 4). The multivariate cox proportional hazard regression model showed significantly higher risk of death in patients with myocardial injury than in those without myocardial injury from symptom onset (hazard ratio, HR, 4.55, 95\% $\mathrm{Cl}, 2.49-8.31, \mathrm{p}<0.001)$ to primary end point, after adjusting for age, sex, preexisting comorbidities, CRP levels, D-dimer levels, NCP types (Table 4). Under this cox regression model, senior age (HR, 2.01, 95\% Cl, 1.03-3.92, P = 0.04), CRP levels (HR, 1.01, 95\% $\mathrm{Cl}, 1.00-1.01, \mathrm{P}=0.001)$ and NCP types $(\mathrm{P}=0.007)$ were other independent risk factors for mortality with COVID-19. In addition, cox regression model with $\mathrm{HsTNI}$ as continuous variable showed that higher HsTNI was also associated with mortality (HR, 3.33 , $95 \% \mathrm{Cl}, 1.96-5.66, \mathrm{P}<0.001)$ (Table S2). 
Table 4

Multivariate Cox Regression Analysis on the Risk Factors Associated with Mortality in Patients With COVID-19 from symptom onset

\begin{tabular}{|c|c|c|c|c|}
\hline \multirow[t]{2}{*}{ Factors } & \multicolumn{2}{|l|}{ Univariate analysis } & \multicolumn{2}{|l|}{ Cox regression model } \\
\hline & Hazard ratio (95\% Cl) & $P$ value & Hazard ratio (95\% Cl) & $P$ value \\
\hline Age, $>65$ years & $3.79(2.32-6.20)$ & $<0.001$ & $2.01(1.03-3.92)$ & 0.04 \\
\hline Sex & $0.53(0.34-0.82)$ & 0.005 & & \\
\hline Hypertension & $2.05(1.34-3.13)$ & 0.001 & & \\
\hline COPD & $2.95(1.64-5.32)$ & $<0.001$ & & \\
\hline Chronic heart disease & $2.16(1.34-3.47)$ & 0.002 & & \\
\hline Cerebrovascular disease & $3.10(1.72-5.59)$ & $<0.001$ & & \\
\hline Myocardial injury & $7.02(4.45-11.08)$ & $<0.001$ & $4.55(2.49-8.31)$ & $<0.001$ \\
\hline CRP & $1.01(1.01-1.02)$ & $<0.001$ & $1.01(1.00-1.01)$ & 0.001 \\
\hline NT-proBNP on admission & $1.00(1.00-1.00)$ & 0.082 & & \\
\hline РCT & $1.00(1.00-1.02)$ & 0.768 & & \\
\hline D-dimer & $1.01(1.00-1.01)$ & 0.003 & & \\
\hline NCP types & & $<0.001$ & & 0.007 \\
\hline severe-common & $3.89(1.95-7.76)$ & $<0.001$ & $2.18(0.92-5.15)$ & 0.075 \\
\hline critically severe-common & $18.42(9.06-37.41)$ & $<0.001$ & $4.33(1.65-11.36)$ & $<0.001$ \\
\hline
\end{tabular}

\section{Discussion}

This study summarizes the clinical characteristics, laboratory, cardiac, and radiographic findings in a large cohort of 304 hospitalized COVID-19 patients, and provides novel information of the prognostic value of pre-existing co-morbidities and myocardial injury. Myocardial injury, senior age, NCP types and CRP levels were independently associated with higher risk of mortality during hospitalization. The myocardial injury was probably associated with inflammation response. The prognostic value of elevated Hs-Tnl in patients with COVID-19 should be of great interest to a broad readership, as a simple blood biomarker test was such a strong predictor of mortality and that sophisticated, expensive and time-consuming cardiac testing with CT and magnetic resonance imaging (MRI) are not needed.

With the high infectivity, COVID-19 has managed to supersede severe acute respiratory syndrome (SARS) in 2003 and Middle East respiratory syndrome (MERS) in 2012 in terms of death toll [13]. The median age was 65 years in our study, greater than previous studies (55.6 and 49 years) [9, 14], mainly due to the severe clinical types and more comorbidities in our patient cohort tending to be older. This is supported by the evidence that the median age of patients is higher in group with myocardial injury than those without (70.5 vs 62.0 years). COVID-19 patients with myocardial injury were prone to develop severe types (77.0\%), and more likely to have pre-existing comorbidities ( $79.2 \%$ vs $58.7 \%$ ), like hypertension, cardiovascular disease and cerebrovascular disease, also confirmed by other studies [9, 15]. Li et.al analyzed six studies involving 1527 COVID-19 patients with previous cardiovascular metabolic diseases, and indicated that this patient group may face a greater risk of developing into the severe condition and that the comorbidities can also greatly affect the prognosis of the COVID-19 [16].

In our study, COVID-19 patients with myocardial injury had obviously elevated myoglobin, and NT-proBNP levels, providing independent corroborating evidence of myocardial injury [17]. In addition, the correlations between Hs-Tnl levels and NT-proBNP 
levels with urea nitrogen levels indicate multiorgan injury along with myocardial injury and worth early monitoring. In prior studies, no significant deviations in coagulation function from the normal range were found but some patients still presented with coagulation dysfunction $[18,19]$. Our study further validated this result, especially in patients with myocardial injury or in critically severe and severe type. Hence, we speculate that coagulation may be not a very important pathophysiological process in all patients with COVID-19. Only some critically ill patients demonstrated abnormal coagulation function and multiple organ dysfunction at the end stage. COVID-19 patients with myocardial injury need active treatments to delay or reverse the progression of disease, supported by the fact that the clinical presentation and the severity of COVID-19, as well as more comorbidities in patients with myocardial injury group. However, patients without myocardial injury received more intravenous immunoglobulin therapy than patients with myocardial injury, and this may be attributable to rapid progression of COVID-19 disease in the later cohort, which makes it too late to use intravenous immunoglobulin therapy.

Two prior studies indicated that there was a strong correlation between myocardial injury and prognosis of COVID-19 patients during hospitalization $[8,9]$. Adding to previous reports, our study further confirmed that myocardial injury, senior age, CRP levels and NCP types are all independent prognostic indicators of mortality in COVID-19 patient. Among these risk factors, myocardial injury, determined by serum Hs-Tnl, was the strongest both as dichotomous and continuous variable. The Hs-Tnl marker can be an ally for earlier identifying myocardial injury, rather than cardiac infarction, thus guiding timely intervention [7]. To date, the exact mechanism of cardiac involvement in COVID-19 remains under investigation. Current studies suggested immune change in patients with MERS [20], SARS [21] and influenza [22], especially changes in peripheral blood T cells, which may contribute to understanding the characteristics, diagnosis, monitoring, prevention and treatment of the disease. Many investigations have already reported that the pathophysiology and outcomes of COVID-19 may be linked to dysregulation of immune response, presenting with lower lymphocyte counts, higher leukocytes counts and significantly reduced CD4 + and CD8 + T lymphocytes levels $[23,24]$. In our study, the CD3, CD4, CD8 counts correlated well with lymphocytes counts and CRP levels, and an elevated inflammation reaction (CRP) and suppressive immune response (CD4+, CD8+) in COVID-19 patients at admission. In addition, traditional cardiovascular risk factors such as diabetes and hyperlipidemia impact immune function, and conversely, dysregulated immunologic status corresponds with elevated risk of incident cardiovascular disease [25]. Another possible mechanism is the direct invasion via angiotensin converting enzyme 2 (ACE2) receptors in cardiovascular system [3]. In our study, we not only found that COVID-19 patients with comorbidities were associated with higher mortality, but also the CRP levels are significantly associated with mortality in multi-variate cox analysis, which indicated there would be inflammation reaction and this was associated with worse outcomes. In addition, IL-6 cytokines were elevated in COVID-19 patients with myocardial injury, justifying the emergence of and association with severity of inflammation, and immune-related markers, like CD3, CD4, CD8 molecules counts and lymphocyte counts were all reduced, more significant in patients with myocardial injury. It was reasonable to presume the invasive coronavirus may dysregulate the immune system, and further lead to severe damage to myocardial tissues. The data from all these studies taken together, suggest that clinicians should consider myocardial injury on presentation, NCP types and inflammation and immune dysregulation what caring for COVID-19 patients. The exact mechanism of myocardial injury merits further investigation, but the findings presented here highlight the prognostic value in identifying myocardial injury with noninvasive biomarker testing on admission in COVID-19 patients and raise the possibility that providers should consider close management of immune response, inflammation and comorbidities in COVID-19 hospitalized patients.

We acknowledge some limitations in our study. First, this was a retrospective, single center study of patients admitted to hospital; multi-center investigations for a larger cohort would be better to assess the clinical characteristics and confirm the outcomes of myocardial injury after infection with COVID-19. Second, because of the logistical limitations at the onset of these emerging infections in Wuhan, some data, such as inflammation biomarker and imaging data were lacked on admission, which limits the further confirmation of potential mechanisms of myocardial injury. Third, the data in this study permit a preliminary assessment of the clinical course and outcomes of patients with COVID-19. The causes of death may involve multiple organ dysfunction in most cases, and it is difficult to differentiate the myocardial injury as the main and direct cause in an individual case. Long-term observation and prospective study design on the effectiveness of treatments specific for the myocardial injury are needed.

\section{Conclusions}


In conclusion, myocardial injury is common in patients hospitalized with COVID-19. Patients with myocardial injury had more severe presentation and complex comorbidities. Furthermore, myocardial injury is independently associated with increased inhospital mortality in patients with COVID-19.

\section{Abbreviations}

COVID-19 = Coronavirus disease 2019; SARS-CoV-2 = severe acute respiratory syndrome coronavirus-2; Hs-Tnl = High-sensitivity troponin $\mathrm{l} ; \mathrm{CK}-\mathrm{MB}$ = creatinine kinase- myocardial band; RT-PCR = real-time reverse-transcriptase polymerase-chain-reaction; $\mathrm{CRP}=$ C-reactive protein; $\mathrm{LDH}=$ lactate dehydrogenase; $\mathrm{PCT}=$ procalcitonin; NT-proBNP = N-terminal pro-B-type natriuretic peptide; $\mathrm{DR}=$ digital radiography; $\mathrm{CT}$ = computed tomography; $\mathrm{NCP}=$ novel coronavirus pneumonia; $\mathrm{MERS}=$ Middle East respiratory syndrome.

\section{Declarations}

\section{Ethics approval and consent to participate:}

This study obtained approval from the Research Ethics Committee of the Renmin Hospital of Wuhan University, Wuhan, China (approval number: WDRY2020-K038). Written consent was obtained from all participants.

\section{Consent for publication:}

Not applicable.

\section{Availability of data and materials:}

The datasets used and/or analysed during the current study are available from the corresponding author on reasonable request.

\section{Competing interests}

The authors declare that they have no competing interests

\section{Funding}

This study was supported by the Construction Research Project of Key Laboratory (Cultivation) of Chinese Academy of Medical Sciences [2019PT310025]; National Natural Science Foundation of China [81971588, 81771811 and 81970331); Capital Clinically Characteristic Applied Research Fund [Z191100006619021]; National Foreign Expert Talent Project[G20190001630]; Education Reform Project of Peking Union Medical College [10023201900204]; Clinical and Translational Fund of Chinese Academy of Medical Sciences [2019XK320063]; Division of Intramural Research, National Heart, Lung and Blood Institute, National Institutes of Health, Department of Health and Human Services. The funding body had no role in the design of the study and collection, analysis, and interpretation of data and in writing the manuscript.

\section{Authors' contributions}

JH, BZ, Quan Z, WY, JX, TL, HZ, ZW, DL, Qing Z, J, CZ: Data curation, Formal analysis; BZ, Quan Z, JZ: Data curation, Resources, Investigation; JH, WY, JX: Writing, Methodology; RW, GL, ZT, AS, HQ, SZ, AA, ML, XZ: Methodology, Project administration, Conceptualization; ML and XZ: Conceptualization, Validation, Supervision, Funding acquisition. All authors have read and approved the manuscript.

\section{Acknowledgements}

Not applicable

\section{References}


1. Organization WH. Pneumonia of unknown cause-China 2020. https://www.who.int/csr/don/05-january-2020-pneumonia-ofunkown-cause-china/en/ (11 July, 2020).

2. Organization WH. Novel coronavirus-China 2020. https://www.who.int/csr/don/12-january-2020-novel-coronaviruschina/en/ (11 July, 2020).

3. Clerkin KJ, Fried JA, Raikhelkar J, Sayer G, Griffin JM, Masoumi A, et al. Coronavirus Disease 2019 (COVID-19) and Cardiovascular Disease. Circulation. 2020. doi:10.1161/circulationaha.120.046941.

4. Organization WH. Coronavirus disease (COVID-19) outbreak. 2020 updated Accessed 11 July 2020. https://covid19.who.int/\# (11 July, 2020).

5. Zhou F, Yu T, Du R, Fan G, Liu Y, Liu Z, et al. Clinical course and risk factors for mortality of adult inpatients with COVID-19 in Wuhan, China: a retrospective cohort study. Lancet. 2020. doi:10.1016/s0140-6736(20)30566-3.

6. Chen N, Zhou M, Dong X, Qu J, Gong F, Han Y, et al. Epidemiological and clinical characteristics of 99 cases of 2019 novel coronavirus pneumonia in Wuhan, China: a descriptive study. Lancet. 2020;395(10223):507-13.

7. Chapman AR, Bularga A, Mills NL. High-Sensitivity Cardiac Troponin Can Be An Ally in the Fight Against COVID-19. Circulation. 2020. doi:10.1161/circulationaha.120.047008.

8. Shi S, Qin M, Shen B, Cai Y, Liu T, Yang F, et al. Association of Cardiac Injury With Mortality in Hospitalized Patients With COVID-19 in Wuhan, China. JAMA Cardiology. 2020. doi:10.1001/jamacardio.2020.0950 \%J JAMA Cardiology.

9. Guo T, Fan Y, Chen M, Wu X, Zhang L, He T, et al. Cardiovascular Implications of Fatal Outcomes of Patients With Coronavirus Disease 2019 (COVID-19). JAMA Cardiology. 2020. doi:10.1001/jamacardio.2020.1017 \% J JMA Cardiology.

10. Guan WJ, Ni ZY, Hu Y, Liang WH, Ou CQ, He JX, et al. Clinical Characteristics of Coronavirus Disease 2019 in China. N Engl J Med. 2020. doi:10.1056/NEJMoa2002032.

11. Thygesen K, Alpert JS, Jaffe AS, Chaitman BR, Bax JJ, Morrow DA, et al. Fourth Universal Definition of Myocardial Infarction (2018) Global heart. 2018;13(4):305-38.

12. National Health Commission of the People's Republic of China. Diagnosis and treatment protocols of pneumonia caused by a novel coronavirus (Revised version 7). Published on March 4. 2020.

https://www.jdzx.net.cn/article/2c907d484df6a9f4014df6c2e8cd0001/2020/3/2c909eaa7074d7280170a3720fcc0351.html (5 April, 2020).

13. Lin L, Lu L, Cao W, Li T. Hypothesis for potential pathogenesis of SARS-CoV-2 infection-a review of immune changes in patients with viral pneumonia. Emerg Microbes Infect. 2020:1-14.doi:10.1080/22221751.2020.1746199.

14. Huang C, Wang Y, Li X, Ren L, Zhao J, Hu Y, et al. Clinical features of patients infected with 2019 novel coronavirus in Wuhan, China. Lancet. 2020;395(10223):497-506.

15. Zhang JJ, Dong X, Cao YY, Yuan YD, Yang YB, Yan YQ, et al. Clinical characteristics of 140 patients infected with SARS-CoV-2 in Wuhan, China. Allergy. 2020. doi:10.1111/all.14238.

16. Li B, Yang J, Zhao F, Zhi L, Wang X, Liu L, et al. Prevalence and impact of cardiovascular metabolic diseases on COVID-19 in China. Clinical research in cardiology: official journal of the German Cardiac Society. 2020. doi:10.1007/s00392-020-01626-9.

17. Zheng YY, Ma YT, Zhang JY, Xie X. COVID-19 and the cardiovascular system. Nature reviews Cardiology. 2020. doi:10.1038/s41569-020-0360-5.

18. Wang D, Hu B, Hu C, Zhu F, Liu X, Zhang J, et al. Clinical Characteristics of 138 Hospitalized Patients With 2019 Novel Coronavirus-Infected Pneumonia in Wuhan, China. Jama. 2020. doi:10.1001/jama.2020.1585.

19. Wu C, Chen X, Cai Y, Xia J, Zhou X, Xu S, et al. Risk Factors Associated With Acute Respiratory Distress Syndrome and Death in Patients With Coronavirus Disease 2019 Pneumonia in Wuhan, China. JAMA Intern Med. 2020. doi:10.1001/jamainternmed.2020.0994.

20. Ko JH, Park GE, Lee JY, Lee JY, Cho SY, Ha YE, et al. Predictive factors for pneumonia development and progression to respiratory failure in MERS-CoV infected patients. J Infect. 2016;73(5):468-75.

21. Li T, Qiu Z, Zhang L, Han Y, He W, Liu Z, et al. Significant changes of peripheral T lymphocyte subsets in patients with severe acute respiratory syndrome. J Infect Dis. 2004;189(4):648-51. 
22. Mohn KG, Cox RJ, Tunheim G, Berdal JE, Hauge AG, Jul-Larsen A, et al. Immune Responses in Acute and Convalescent Patients with Mild, Moderate and Severe Disease during the 2009 Influenza Pandemic in Norway. PloS one.

2015;10(11):e0143281.

23. Mehta P, McAuley DF, Brown M, Sanchez E, Tattersall RS, Manson JJ. COVID-19: consider cytokine storm syndromes and immunosuppression. The Lancet. 2020;395(10229):1033-4.

24. Qin C, Zhou L, Hu Z, Zhang S, Yang S, Tao Y, et al. Dysregulation of immune response in patients with COVID-19 in Wuhan, China. Clin Infect Dis. 2020;71(15):762-8. doi:10.1093/cid/ciaa248.

25. Driggin E, Madhavan MV, Bikdeli B, Chuich T, Laracy J, Bondi-Zoccai G, et al. Cardiovascular Considerations for Patients, Health Care Workers, and Health Systems During the Coronavirus Disease 2019 (COVID-19) Pandemic. J Am Coll Cardiol. 2020. doi:10.1016/j.jacc.2020.03.031.

\section{Figures}
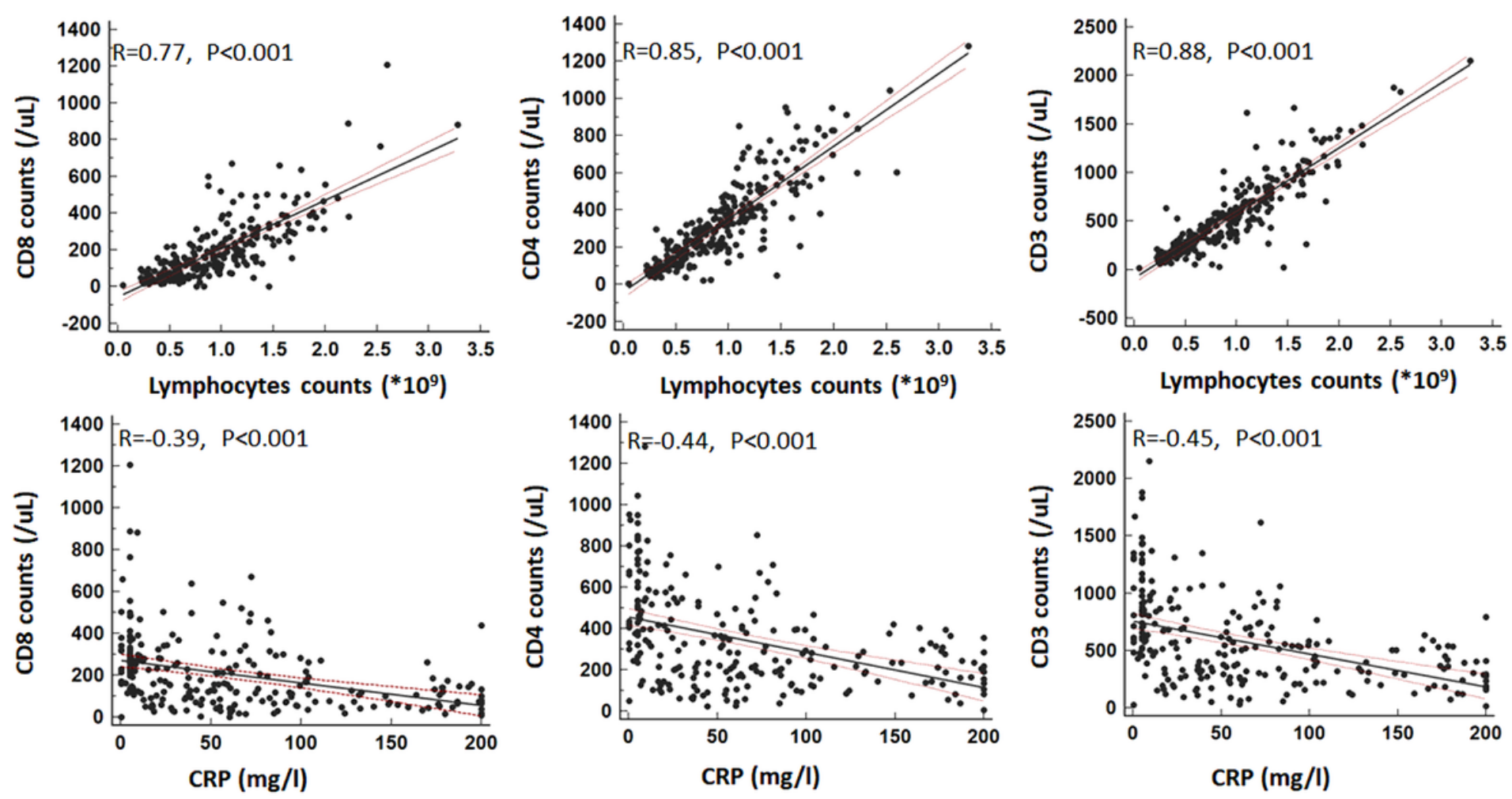

Figure 1

Associations between CD3, CD4, CD8 and lymphocytes biomarkers Strong correlations were found between CD3, CD4, CD8 and lymphocytes biomarkers. CD, Cluster of Differentiation of T cells. 

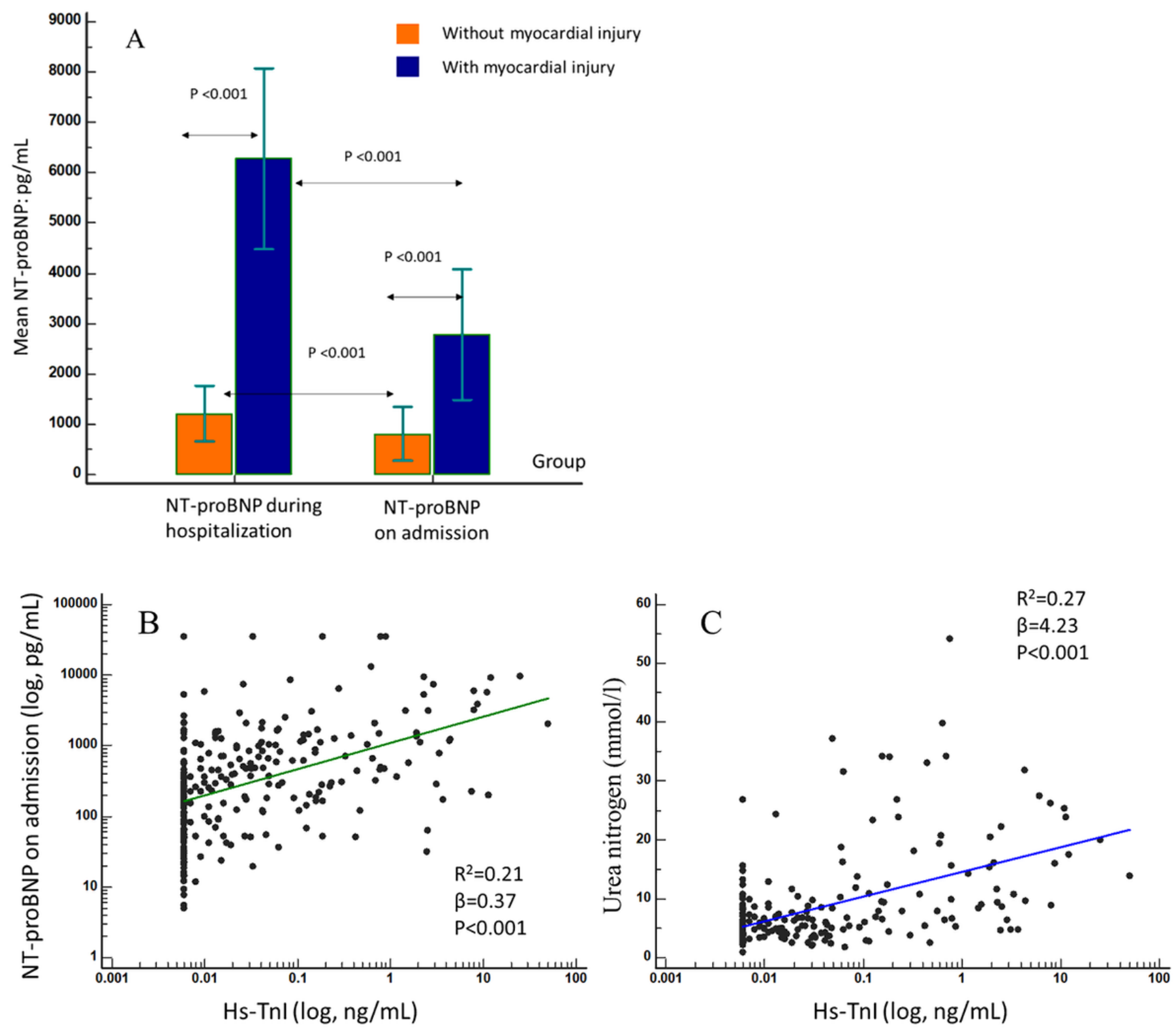

Figure 2

Progression of NT-proBNP of COVID-19 patients and correlation Between Serum high-sensitivity Tnl and NT-proBNP With Urea nitrogen. The mean NT-proBNP progression of COVID patients on admission and during hospitalization. NT-proBNP, Nterminal proB-type natriuretic peptide, Hs-Tnl: high-sensitivity troponin I. when High-sensitivity troponin I levels $<0.006 \mathrm{ng} / \mathrm{mL}$, it was recorded as $0.006 \mathrm{ng} / \mathrm{mL}$ at convenience. 


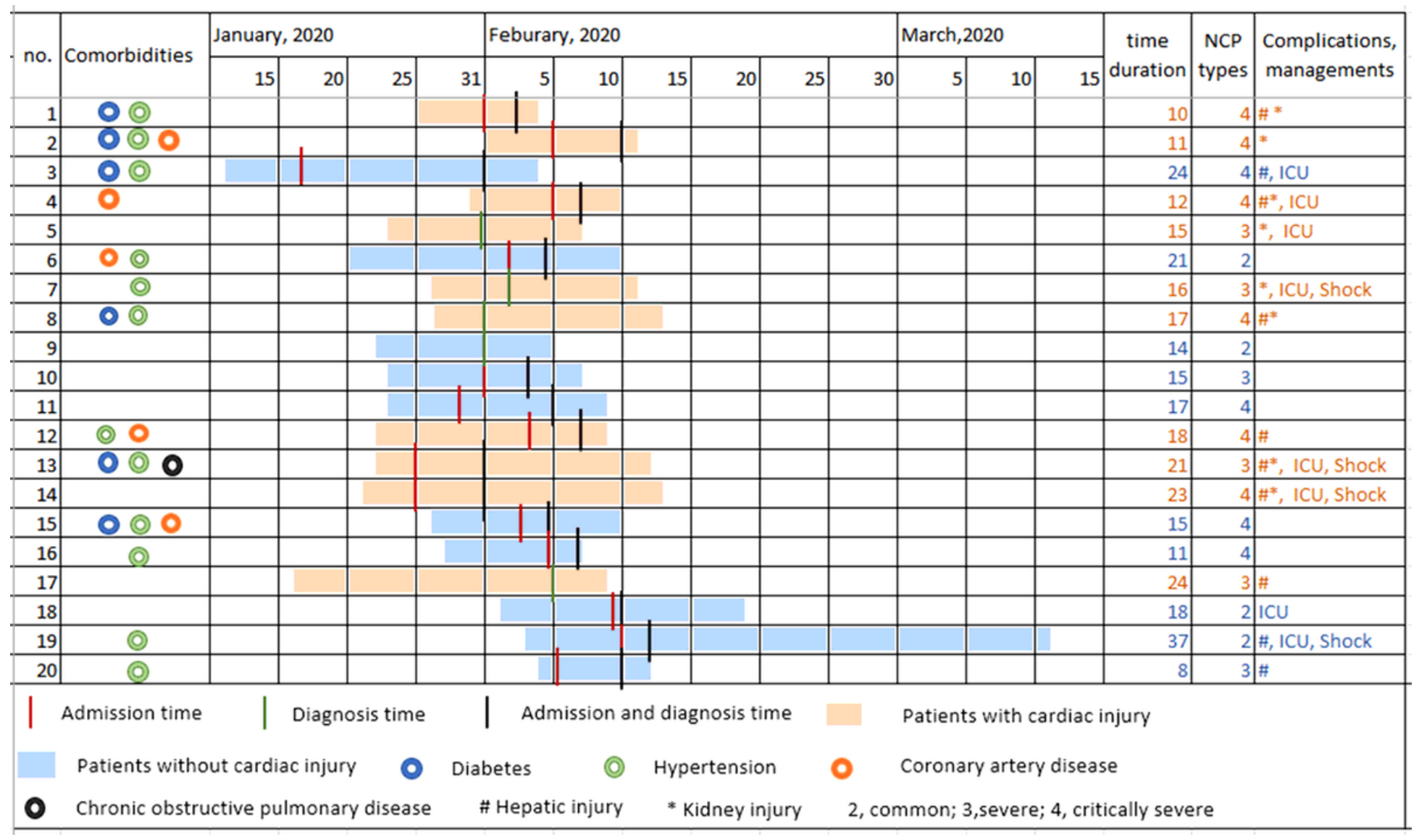

\section{Figure 3}

Days from symptom onset to death of COVID-19 patients with or without myocardial injury and their comorbidities, complications, managements. Comparison of days from symptom onset to death of typical 20 patients with COVID-19 with myocardial injury (10 patients) or without myocardial injury (10 patients) randomly selected from dead patients. Patients with myocardial injury were more likely to have comorbidities, severe presentation, and complications, shock and ICU managements. However, no significant difference was found for time duration from symptom onset to death in two groups. This figure tells us that myocardial injury may be associated with more severe presentation. ICU: intensive care unit. 


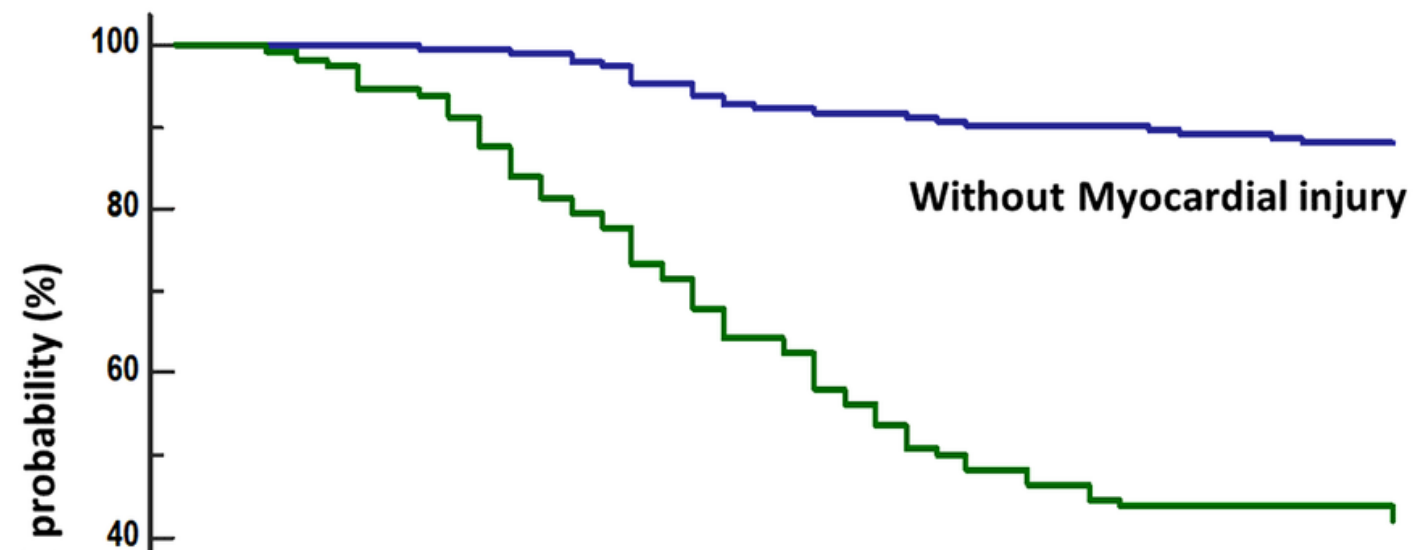

With Myocardial injury

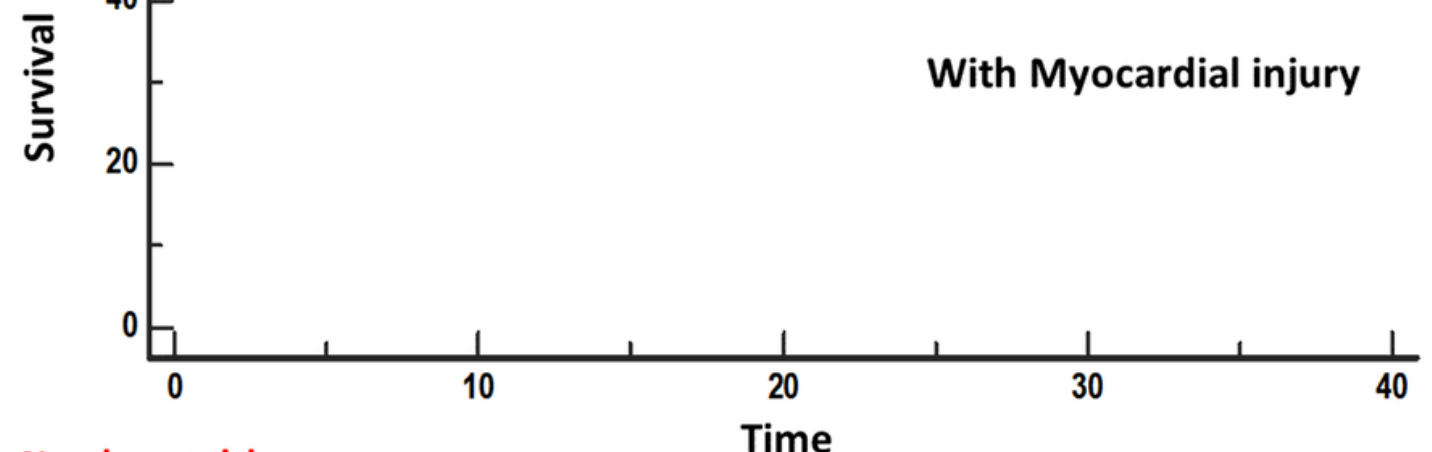

Number at risk

$\begin{array}{llllll}\text { Without Myocardial injury } & 192 & 191 & 177 & 173 & 169 \\ \text { With Myocardial injury } & 112 & 98 & 70 & 50 & 47\end{array}$

Figure 4

Mortality During Hospitalization Between Patients With vs Without Myocardial injury. Kaplan-Meier survival curves for mortality from symptom onset to follow-up date.

\section{Supplementary Files}

This is a list of supplementary files associated with this preprint. Click to download.

- SupplementaryFiles.docx 